\title{
TURBO DETECTION OF SYMBOL-BASED NON-BINARY LDPC-CODED SPACE-TIME SIGNALS USING SPHERE PACKING MODULATION
}

\author{
O. Alamri, F. Guo, M. Jiang and L. Hanzo* \\ School of Electr. and Comp. Sc., Univ. of Southampton, SO17 1BJ, UK. \\ Tel: +44-2380-593 125, Fax: +44-2380-593045 \\ Email: 1h@ecs.soton.ac.uk \\ http: //www-mobile.ecs.soton.ac.uk
}

\begin{abstract}
A recently proposed space-time signal construction method that combines orthogonal design with sphere packing, referred to here as (STBC-SP), has shown useful performance improvements over Alamouti's conventional orthogonal design. As a further advance, non-binary LDPC codes have been capable of attaining substantial performance improvements over their binary counterparts. In this paper, we demonstrate that the performance of STBC-SP systems can be further improved by concatenating sphere packing aided modulation with non-binary LDPC codes and performing symbolbased turbo detection. We present simulation results for the proposed scheme communicating over a correlated Rayleigh fading channel. At a $B E R$ of $10^{-6}$, the proposed symbolbased turbo-detected STBC-SP scheme was capable of achieving a coding gain of approximately $26.6 \mathrm{~dB}$ over the identicalthroughput 1 bit/symbol uncoded STBC-SP benchmarker scheme. The proposed scheme also achieved a coding gain of approximately $3 d B$ at a $B E R$ of $10^{-6}$ over a recently proposed bit-based turbo-detected STBC-SP benchmarker scheme.
\end{abstract}

\section{INTRODUCTION}

The adverse effects of channel fading may be significantly reduced by employing space-time coding invoking multiple antennas [1]. Alamouti [2] discovered an appealingly simple transmit diversity scheme employing two transmit antennas. This low-complexity design inspired Tarokh et al. $[3,4]$ to generalise Alamouti's transmit diversity scheme using the principle of orthogonal design to an arbitrary number of transmit antennas. Since then, the pursuit of designing better space-time modulation schemes has attracted considerable further attention [2]. The concept of combining orthogonal transmit diversity designs with the principle of sphere packing was introduced by $\mathrm{Su}$ et al. in [5]. Orthogonal transmit diversity designs can be described recursively [6] as follows. Let $G_{1}\left(x_{1}\right)=x_{1} I_{1}$, and

$$
\begin{aligned}
& G_{2^{k}}\left(x_{1}, \ldots, x_{k+1}\right) \\
= & {\left[\begin{array}{cc}
G_{2^{k-1}}\left(x_{1}, \ldots, x_{k}\right) & x_{k+1} I_{2^{k-1}} \\
-x_{k+1}^{*} I_{2^{k-1}} & G_{2^{k-1}}^{\mathcal{H}}\left(x_{1}, \ldots, x_{k}\right)
\end{array}\right], }
\end{aligned}
$$

for $k=1,2,3, \ldots$,where $x_{k+1}^{*}$ is the complex conjugate of $x_{k+1}$, $G_{2^{k-1}}^{\mathcal{H}}\left(x_{1}, \ldots, x_{k}\right)$ is the Hermitian of $G_{2^{k-1}}\left(x_{1}, \ldots, x_{k}\right)$ and $I_{2^{k-1}}$ is a $\left(2^{k-1} \times 2^{k-1}\right)$ identity matrix. Then, $G_{2^{k}}\left(x_{1}, x_{2}, \ldots\right.$, $\left.x_{k+1}\right)$ constitutes an orthogonal design of size $\left(2^{k} \times 2^{k}\right)$, which maps the complex variables representing $\left(x_{1}, x_{2}, \ldots, x_{k+1}\right)$ to $2^{k}$ transmit antennas. In other words, $x_{1}, x_{2}, \ldots, x_{k+1}$ represent

*The financial support of the European Union and the Ministry of Higher Education of Saudi Arabia is gratefully acknowledged $k+1$ complex modulated symbols to be transmitted from $2^{k}$ transmit antennas in $T=2^{k}$ time slots. It was shown in [5] that the diversity product (coding advantage ${ }^{1}$ ) of an orthogonal transmit diversity scheme is determined by the minimum Euclidean distance of the vectors $\left(x_{1}, x_{2}, \ldots, x_{k+1}\right)$. Therefore, in order to maximise the achievable coding advantage, it was proposed in [5] to use sphere packing schemes that have the best known minimum Euclidean distance in the $2(k+1)$-dimensional real-valued Euclidean space $R^{2(k+1)}$ [7]. The results of [5] demonstrated that the proposed Sphere Packing (SP) aided Space-Time Block Coded (STBC) system of Section 2, referred to here as STBC-SP, was capable of outperforming the conventional orthogonal design based STBC schemes of $[2,3]$.

The authors of [8] proposed a novel system that exploits the advantages of both iterative demapping and decoding [9] as well as those of the STBC-SP scheme of [5]. The STBC-SP demapper of [5] was modified in [8] for the sake of accepting the a priori information passed to it from the channel decoder as extrinsic information.

Surprisingly, the family of Low Density Parity Check (LDPC) codes originally devised by Gallager as early as 1963 [10] remained more or less unexploited until after the discovery of turbo codes in 1993 [11]. Since then, however, LDPC codes have experienced a renaissance [12] and attracted substantial research interests. MacKay and Neal demonstrated in [13] that despite their simple decoding structure, LDPC codes are also capable of operating near the channel capacity. Richardson [14] suggested the employment of a differential belief propagation decoding algorithm for binary LDPC codes using the Fast Fourier Transform (FFT) for reducing the decoding complexity imposed. In 1998, Davey and MacKay proposed a non-binary version of LDPC codes [15], which was potentially capable of outperforming binary LDPC codes. When using Richardson's FFT-based decoding algorithm [14], the complexity of non-binary LDPCs increases only linearly with respect to the size of the associated Galois field.

Motivated by the performance improvements reported in [5] and [15], we propose a novel symbol-based iterative system that exploits the advantages of both non-binary LDPC codes [15] as well as those of the STBC-SP scheme of [5]. Given a certain effective throughput, our simulation results demonstrate that the proposed turbo detection aided STBC-SP scheme is capable of providing attractive performance improvements over established orthogonal STBC designs, constituted by the STBC-SP scheme of [5] as well as over the bit-based turbo-detected STBC-SP scheme of [8].

This paper is organised as follows. In Section 2, a brief description of orthogonal design with sphere packing modulation is pre-

\footnotetext{
${ }^{1}$ The diversity product or coding advantage was defined as the estimated gain over an uncoded system having the same diversity order as the coded system [5].
} 
sented, followed by a brief system overview in Section 3. Symbolbased iterative decoding is described in Section 4. Simulation results and our discussions are provided in Section 5. Finally, we conclude in Section 6.

\section{ORTHOGONAL DESIGN WITH SPHERE PACKING MODULATION}

In this contribution, space-time systems employing two transmit antennas are considered, where the space-time signal is given by [2]

$$
G_{2}\left(x_{1}, x_{2}\right)=\left[\begin{array}{cc}
x_{1} & x_{2} \\
-x_{2}^{*} & x_{1}^{*}
\end{array}\right],
$$

where the rows and columns of Equation (1) represent the temporal and spatial dimensions, corresponding to two consecutive time slots and two transmit antennas, respectively. According to Alamouti's design [2] for example, $x_{1}$ and $x_{2}$ represent conventional BPSK modulated symbols transmitted in the $1^{\text {st }}$ and $2^{\text {nd }}$ time slots and no effort is made to jointly design a signal constellation for the various combinations of $x_{1}$ and $x_{2}$. For the sake of generalising our treatment, let us assume that there are $L$ legitimate space-time signals $G_{2}\left(x_{l, 1}, x_{l, 2}\right), l=0,1, \ldots, L-1$, where $L$ represents the number of sphere-packed modulated symbols. The transmitter, then, has to choose the modulated signal from these $L$ legitimate symbols, which have to be transmitted over the two antennas in two consecutive time slots, where the throughput of the system is given by $\left(\log _{2} L\right) / 2$ bits per channel use. In contrast to Alamouti's independent design of the two time slots' signals, our aim is to design $x_{l, 1}$ and $x_{l, 2}$ jointly, such that they have the best minimum Euclidean distance from all other $(L-1)$ legitimate transmitted space-time signals, since this minimises the system's error probability. Let $\left(a_{l, 1}, a_{l, 2}, a_{l, 3}, a_{l, 4}\right), l=0,1, \ldots, L-1$, be phasor points from the four-dimensional real-valued Euclidean space $\mathrm{R}^{4}$, where each of the four elements $a_{l, 1}, a_{l, 2}, a_{l, 3}, a_{l, 4}$ gives one coordinate of the two time-slots' complex-valued phasor points. Hence, $x_{l, 1}$ and $x_{l, 2}$ may be written as

$$
\begin{aligned}
\left\{x_{l, 1}, x_{l, 2}\right\} & =T\left(a_{l, 1}, a_{l, 2}, a_{l, 3}, a_{l, 4}\right) \\
& =\left\{a_{l, 1}+j a_{l, 2}, a_{l, 3}+j a_{l, 4}\right\} .
\end{aligned}
$$

In the four-dimensional real-valued Euclidean space $\mathrm{R}^{4}$, the lattice $D_{4}$ is defined as a sphere packing having the best minimum Euclidean distance from all other $(L-1)$ legitimate constellation points in $\mathrm{R}^{4}$ [7]. More specifically, $D_{4}$ may be defined as a lattice that consists of all legitimate sphere packed constellation points having integer coordinates $\left[\begin{array}{llll}a_{1} & a_{2} & a_{3} & a_{4}\end{array}\right]$ uniquely and unambiguously describing the legitimate combinations of the two time-slots' modulated symbols in Alamouti's scheme, but subjected to the sphere packing constraint of $a_{1}+a_{2}+a_{3}+a_{4}=k$, where $k$ is an even integer. Assuming that $S=\left\{s^{l}=\left[a_{l, 1}, a_{l, 2}, a_{l, 3}, a_{l, 4}\right]\right.$ $\left.\in \mathrm{R}^{4}: 0 \leq l \leq L-1\right\}$ constitutes a set of $L$ legitimate constellation points from the lattice $D_{4}$ having a total energy of $E \triangleq$ $\sum_{l=0}^{L-1}\left(\left|a_{l, 1}\right|^{2}+\left|a_{l, 2}\right|^{2}+\left|a_{l, 3}\right|^{2}+\left|a_{l, 4}\right|^{2}\right)$, and upon introducing the notation

$$
C_{l}=\sqrt{\frac{2 L}{E}} G_{2}\left(x_{l, 1}, x_{l, 2}\right), \quad l=0,1, \ldots, L-1,
$$

we have a set of space-time signals, $\left\{C_{l}: 0 \leq l \leq L-1\right\}$, whose diversity product is determined by the minimum Euclidean distance of the set of $L$ legitimate constellation points in $\mathrm{S}$.

\section{SYSTEM OVERVIEW}

The schematic of the entire system is shown in Figure 1. The source bits are encoded by a non-binary LDPC encoder [15], and
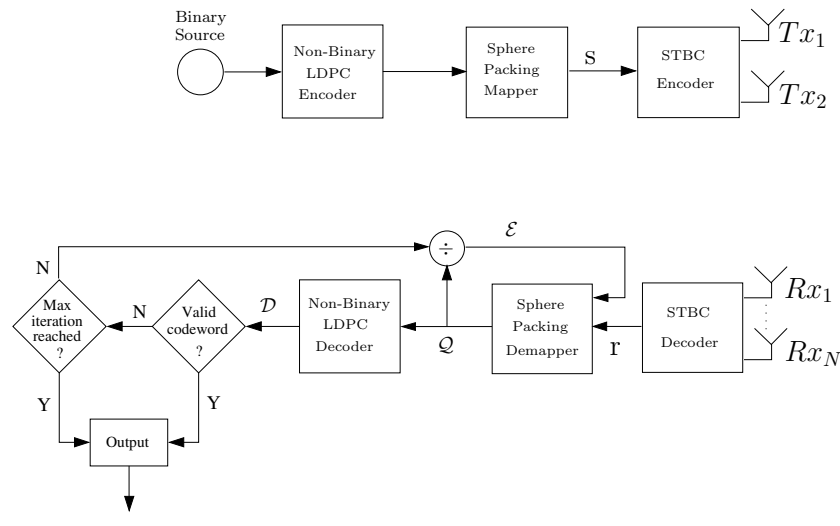

Figure 1: System schematic

each LDPC encoded symbol is mapped to the corresponding sphere packing modulated symbol $s^{l} \in S, 0 \leq l \leq L-1$. There is a natural one-to-one mapping between the non-binary LDPC code used and the non-binary sphere-packing scheme, allowing us to create purely symbol-based system. The STBC encoder then maps the sphere packing modulated symbol $s^{l}$ to a space-time signal $C_{l}=\sqrt{\frac{2 L}{E}} G_{2}\left(x_{l, 1}, x_{l, 2}\right), 0 \leq l \leq L-1$, using Equation (2). Subsequently, each sphere-packed space-time coded symbol is transmitted over $T=2$ time slots using two transmit antennas, as shown in Equation (1).

In this treatise, we considered a correlated narrowband Rayleigh fading channel, associated with a normalised Doppler frequency of $f_{D}=0.1$. The complex fading envelope is assumed to be constant across the transmission period of a sphere-packed space-time coded symbol spanning $T=2$ time slots. The complex Additive White Gaussian Noise (AWGN) of $n=n_{I}+j n_{Q}$ is also added to the received signal, where $n_{I}$ and $n_{Q}$ are two independent zero mean Gaussian random variables having a variance of $\sigma_{n}^{2}=\sigma_{n_{I}}^{2}=\sigma_{n_{Q}}^{2}=N_{0} / 2$ per dimension, with $N_{0} / 2$ representing the double-sided noise power spectral density expressed in $\mathrm{W} / \mathrm{Hz}$.

As shown in Figure 1, the received complex-valued symbols are first decoded by the STBC decoder to produce a received spherepacked symbol $r$, which is fed into the sphere packing demapper, where the soft-metric $\mathcal{Q}\left(s_{k}\right)$ is calculated. More explicitly, the notation $\mathcal{Q}\left(s_{k}\right)$ represents the soft metric passed from the sphere packing demapper to the LDPC decoder based on the symbol probability of the $k^{t h}$ symbol of the LDPC codeword. As seen in Figure 1 , these soft-metrics are passed as a-priori information to the LDPC decoder, which carries out a specified number of LDPC iterations and produces the decoded a-posteriori probability $\mathcal{D}$. Based on the a-posteriori probability, a tentative hard decision will be made and the resultant codeword will be checked by the LDPC code's parity check matrix. If the resultant vector is an allzero sequence, then a legitimate codeword has been found, and the hard-decision based sequence will be output. Otherwise, if the maximum affordable number of iterations has not been reached, the a-priori information, $\mathcal{Q}$, is removed from the a-posteriori probability denoted by $\mathcal{D}$ using symbol-based element-wise division, as will be shown in Section 4, and fed back to the demodulator for the next iteration, as seen in Figure 1. This process continues, until the affordable maximum number of iterations has been encountered or a legitimate codeword has been found. 


\section{SYMBOL-BASED ITERATIVE LDPC AND SPHERE-PACKING DECODING}

For the sake of simplicity, a system having a single receive antenna is considered, although its extension to systems having more than one receive antenna is straightforward. Assuming perfect channel estimation, the complex-valued channel output symbols received during two consecutive time slots are first diversity-combined in order to extract the estimates $\tilde{x}_{1}$ and $\tilde{x}_{2}$ of the most likely transmitted symbols $x_{l, 1}$ and $x_{l, 2}$ [2][1, pp.400-401], resulting in

$$
\begin{aligned}
& \tilde{x}_{1}=\left(\left|h_{1}\right|^{2}+\left|h_{2}\right|^{2}\right) \cdot x_{l, 1}+\dot{w} \\
& \tilde{x}_{2}=\left(\left|h_{1}\right|^{2}+\left|h_{2}\right|^{2}\right) \cdot x_{l, 2}+\dot{w}
\end{aligned}
$$

where $h_{1}$ and $h_{2}$ represent the Channel Impulse Response (CIR) corresponding to the first and second transmit antennas, respectively, and $w$ is a zero-mean complex Gaussian random variable with variance $\sigma_{w}^{2}=\left(\left|h_{1}\right|^{2}+\left|h_{2}\right|^{2}\right) \cdot \sigma_{n}^{2}$. A received spherepacked symbol $r$ is then constructed from the estimates $\tilde{x}_{1}$ and $\tilde{x}_{2}$ using Equation (2) as

$$
r=T^{-1}\left(\tilde{x}_{1}, \tilde{x}_{2}\right)
$$

where $r=\left\{\left[\tilde{a}_{1}, \tilde{a_{2}}, \tilde{a_{3}}, \tilde{a_{4}}\right] \in R^{4}\right\}$. The received sphere-packed symbol $r$ can be written as

$$
r=h \cdot \sqrt{\frac{2 L}{E}} \cdot s^{l}+w
$$

where $h=\left(\left|h_{1}\right|^{2}+\left|h_{2}\right|^{2}\right), s^{l} \in S, 0 \leq l \leq L-1$, and $w$ is a four-dimensional Gaussian random variable having a variance of $\sigma_{w}^{2}=\sigma_{w}^{2}=h \cdot \sigma_{n}^{2}$, since the symbol constellation $S$ is fourdimensional. Additionally, there is a one-to-one mapping between $l$ and the elements from the $G F(q)$ of the non-binary LDPC code, such that $L=q$. According to Equation (7), the conditional PDF $P\left(r \mid s^{l}\right)$ is given by

$$
\begin{aligned}
P\left(r \mid s^{l}\right) & =\frac{1}{\left(2 \pi \sigma_{w}^{2}\right)^{\frac{N_{D}}{2}}} e^{-\frac{1}{2 \sigma_{w}^{2}}\left(r-\alpha \cdot s^{l}\right)^{2}}, \\
& =\frac{1}{\left(2 \pi \sigma_{w}^{2}\right)^{\frac{N_{D}}{2}}} e^{-\frac{1}{2 \sigma_{w}^{2}}\left(\sum_{i=1}^{4}\left(\tilde{a}_{i}-\alpha \cdot a_{l, i}\right)^{2}\right)},
\end{aligned}
$$

where we have $\alpha=h \cdot \sqrt{\frac{2 L}{E}}$ and $N_{D}=4$, since a four-dimensional sphere-packed symbol constellation is used.

Similarly, the conditional PDF $P\left(s^{l} \mid r\right)$ is given by

$$
P\left(s^{l} \mid r\right)=\frac{P\left(r \mid s^{l}\right) \cdot P\left(s^{l}\right)}{P(r)}
$$

Assuming that the LDPC codeword consists of $K G F(q)$ symbols, the sphere packing demapper of Figure 1 will process $K$ received sphere-packed symbols, $\left(r_{0}, r_{1}, \ldots, r_{K-1}\right)$, at a time to produce the following soft-metric $(K \times L)$ matrix using Equation (9)

$$
\mathcal{Q}=\left[\begin{array}{llll}
\mathcal{Q}\left(s_{0}\right) & \mathcal{Q}\left(s_{1}\right) & \cdots & \mathcal{Q}\left(s_{K-1}\right)
\end{array}\right]^{T},
$$

where,

$$
\begin{aligned}
& \mathcal{Q}\left(s_{k}\right) \\
= & {\left[\begin{array}{llll}
P\left(s_{k}=s^{0} \mid r_{k}\right) & P\left(s_{k}=s^{1} \mid r_{k}\right) & \cdots & P\left(s_{k}=s^{L-1} \mid r_{k}\right)
\end{array}\right], }
\end{aligned}
$$

for $k=0,1, \ldots, K-1$. All the probabilities corresponding to a specific row in $\mathcal{Q}$, which correspond to a specific received symbol, should be normalised so that they sum up to unity. During the first iteration, $P\left(s^{l}\right), 0 \leq l \leq L-1$, in Equation (9) has to be set to $1 / q$, since no a-prior $i$ information is yet available from the LDPC decoder.

The soft-metric matrix $\mathcal{Q}$ of Equation (10) is then fed into the LDPC decoder of Figure 1, as a-priori knowledge, which produces a decoded a-posteriori probability matrix, $\mathcal{D}$, of size $(K \times$ $L)$. The a-priori knowledge fed into the LDPC decoder of Figure 1 is removed from the decoded a-posteriori probability matrix, $\mathcal{D}$, by symbol-based element-wise division [16] for the sake of passing over the extrinsic information back to the sphere packing demapper for further iterations as alluded to before. More specifically, the following $(K \times L)$ extrinsic information matrix is constructed

$\mathcal{E}=\left[\begin{array}{cccc}P\left(s_{0}=s^{0}\right) & P\left(s_{0}=s^{1}\right) & \cdots & P\left(s_{0}=s^{L-1}\right) \\ P\left(s_{1}=s^{0}\right) & P\left(s_{1}=s^{1}\right) & \cdots & P\left(s_{1}=s^{L-1}\right) \\ \vdots & \vdots & \ddots & \vdots \\ P\left(s_{K-1}=s^{0}\right) & P\left(s_{K-1}=s^{1}\right) & \cdots & P\left(s_{K-1}=s^{L-1}\right)\end{array}\right]$

where we have

$P\left(s_{k}=s^{l}\right)=\frac{d_{k}^{l}}{q_{k}^{l}}, \quad 0 \leq l \leq L-1 \quad$ and $\quad 0 \leq k \leq K-1$,

and $d_{k}^{l}$ as well as $q_{k}^{l}$ refer to the elements at the cross-over point of the $k^{t h}$ row and $l^{t h}$ column of matrices $\mathcal{D}$ and $\mathcal{Q}$, respectively. Again, the probabilities corresponding to a specific row of $\mathcal{E}$ should be normalised so that they add up to unity. The STBCSP demapper exploits the extrinsic information, $\mathcal{E}$, for the sake of providing an improved soft-metric, $\mathcal{Q}$, which is passed to the LDPC decoder of Figure 1 for further iterations, until a legitimate codeword is found or the affordable maximum number of iterations has been exhausted.

\section{RESULTS AND DISCUSSION}

Without loss of generality, we considered a sphere packing modulation scheme associated with $L=16$ using two transmit and a single receiver antenna in order to demonstrate the performance improvements achieved by the proposed system. All simulation parameters are listed in Table 1.

Since the space-time signal, which is constructed from an orthogonal design using the sphere packing scheme of Equation (3) is multiplied by a factor that is inversely proportional to $\sqrt{E}$, namely by $\sqrt{\frac{2 L}{E}}$, it is desirable to choose a specific subset of $L=16$ points from the entire set of legitimate constellation points hosted by $D_{4}$, which results in the minimum total energy. It was shown in [7] that there is a total of 24 legitimate symbols ${ }^{2}$ hosted by $D_{4}$ having an identical minimum energy of $E=2$. We used a computer search for determining the optimum choice of the $L=16$ points out of the possible 24 points, which possess the highest minimum Euclidean distance, hence minimising the error probability.

Figure 2 compares the performance of the proposed symbolbased non-binary LDPC-coded STBC-SP scheme against that of an identical-throughput 1 Bit Per Symbol (1BPS) uncoded STBCSP scheme [5], to the conventional orthogonal STBC design based $[2,3]$ schemes and against a recently proposed bit-based convolutional-coded turbo-detected STBC-SP scheme [8]. These comparison were carried out when communicating over a correlated

${ }^{2}$ In simple terms, the sphere centred at $(0,0,0,0)$ has 24 spheres around it, centred at the points $(+/-1,+/-1,0,0)$, where any choice of signs and any ordering of the coordinates is legitimate [7, p.9]. 


\begin{tabular}{|l|r|}
\hline Modulation & Sphere Packing with $L=16$ \\
\hline No. of Transmitters & 2 \\
\hline No. of Receivers & 1 \\
\hline Channel & Correlated Rayleigh Fading \\
\hline $\begin{array}{l}\text { Normalised Doppler } \\
\text { frequency }\end{array}$ & 0.1 \\
\hline $\begin{array}{l}\text { Average LDPC } \\
\text { column weight }\end{array}$ & 2.5 \\
\hline LDPC coding rate & 0.5 \\
\hline LDPC decoding field & $G F(16)$ \\
\hline System throughput & 1 bit/symbol \\
\hline Coded Blocklength & 6000 bits \\
\hline
\end{tabular}

Table 1: System parameters

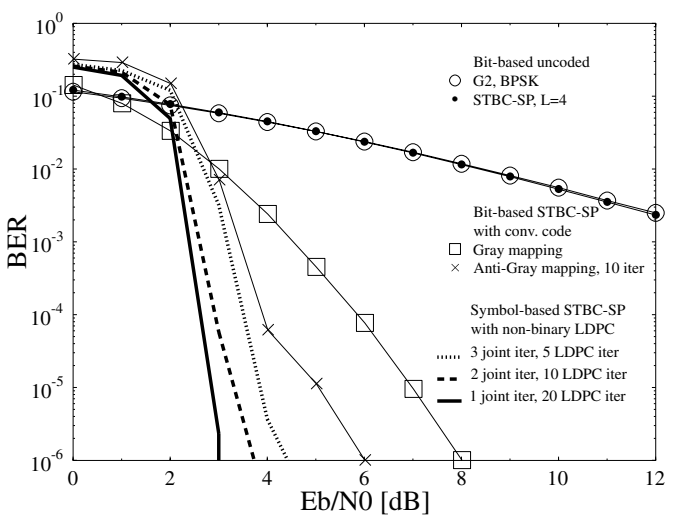

Figure 2: Performance comparison of various symbol-based non-binary LDPC-coded STBC-SP schemes in conjunction with the parameters listed in Table 1 against an identical-throughput 1BPS uncoded STBC-SP scheme [5], Alamouti's conventional $G_{2}$-BPSK scheme [2] and against bit-based convolutional-coded STBC-SP schemes [8] employing Gray Mapping (GM) and Anti-Gray Mapping (AGM), when communicating over a correlated Rayleigh fading channel having $f_{D}=0.1$.

Rayleigh fading channel having a normalised Doppler frequency of $f_{D}=0.1$ and using the simulation parameters listed in Table 1 . More explicitly, Figure 2 demonstrates that a coding advantage of about $26.6 \mathrm{~dB}$ was achieved at a BER of $10^{-6}$ by the symbolbased non-binary LDPC-coded STBC-SP scheme over both the uncoded STBC-SP [5] and the conventional orthogonal STBC design based $[2,3]$ schemes for transmission over the correlated Rayleigh fading channel considered. Additionally, a coding advantage of approximately $5 d B$ and $3 d B$ were attained over the recently proposed 1BPS-throughput convolutional-coded bit-based STBCSP schemes [8] employing Gray Mapping (GM) and Anti-Gray Mapping (AGM) [9], respectively.

Figure 3 compares the performance of the proposed scheme when $I_{L D P C}=3 \mathrm{LDPC}$ iterations are used in conjunction with various numbers of joint iterations against both that of an identicalthroughput 1 Bit Per Symbol (1BPS) uncoded STBC-SP scheme [5], conventional orthogonal STBC design based $[2,3]$ schemes and the bit-based convolutional-coded turbo-detected STBC-SP scheme of [8], when communicating over a correlated Rayleigh fading channel having a normalised Doppler frequency of $f_{D}=0.1$ and using the simulation parameters listed in Table 1. It is evident from Figure 3 that no significant coding gain is attained after encountering $I_{\text {joint }}=2$ or 3 joint iterations, when using $I_{L D P C}=3$

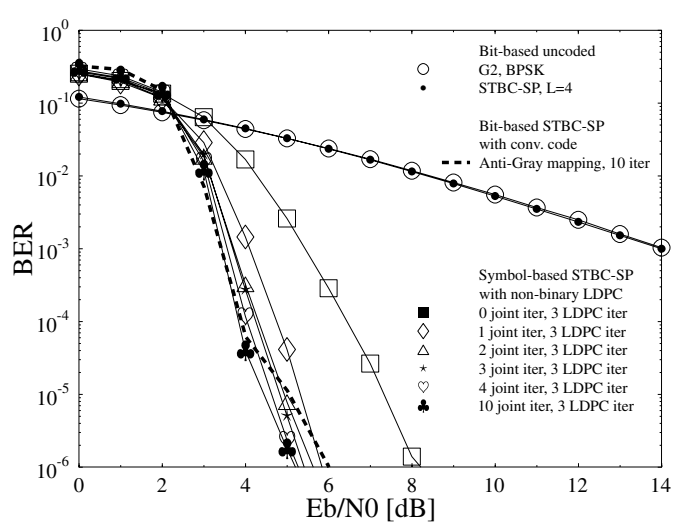

Figure 3: Performance comparison of symbol-based non-binary LDPCcoded STBC-SP schemes using a fixed number of LDPC iterations and various numbers of joint iterations against both an identical-throughput 1BPS uncoded STBC-SP scheme [5], Alamouti's conventional $G_{2^{-}}$ BPSK scheme [2] and against bit-based convolutional-coded STBC-SP schemes [8] employing Gray Mapping (GM) and Anti-Gray Mapping (AGM), when communicating over a correlated Rayleigh fading channel having $f_{D}=0.1$.

LDPC iterations. Similarly, Figure 4 compares the performance of the proposed scheme against all schemes considered in Figure 3, when a maximum of $I_{\text {joint }}=3$ joint iterations are encountered in conjunction with various numbers of LDPC iterations. In contrast to the observation made in the context of Figure 3, considerable performance improvements can be attained, when using a specific maximum number of joint iterations, by increasing the number of inner LDPC iterations, as illustrated in Figure 4.

Figure 5 shows the achievable coding gain of various symbolbased non-binary LDPC-coded STBC-SP schemes in conjunction with different combinations of external joint and internal LDPC iterations, as compared to both the identical-throughput 1BPS uncoded STBC-SP scheme of [5] and to Alamouti's conventional $G_{2}$-BPSK scheme [2], when communicating over a correlated Rayleigh fading channel having $f_{D}=0.1$. The horizontal dotted line corresponds to the coding gain of the bit-based convolutionalcoded STBC-SP scheme of [8] employing anti-Gray mapping and after $I=10$ iterations as compared to both the identicalthroughput 1BPS uncoded STBC-SP scheme of [5] and to Alamouti's conventional $G_{2}$-BPSK scheme [2]. It can be observed from Figure 5 that a considerable coding gain can be obtained at a specific number of external joint iterations upon increasing the number of LDPC iterations. This statement is valid even for a high number of external joint iterations. For example, it is illustrated in Figure 5 that a coding gain advantage of about $4 d B$ can be attained $I_{\text {joint }}=10$ joint iterations upon increasing the number of LDPC iterations from $I_{L D P C}=1$ to 20 . However, the attainable coding gain improvement becomes less significant, when the number of external joint iterations is increased, while maintaining the number of inner LDPC iterations, especially when $I_{L D P C}=3$ or more LDPC iterations are used. This leads to a general design rule of thumb for the proposed scheme, which implies that no more than $I_{\text {joint }}=3$ joint iterations are recommended, since it is more beneficial to increase the number of LDPC iterations for the sake of maximising the attainable performance. 


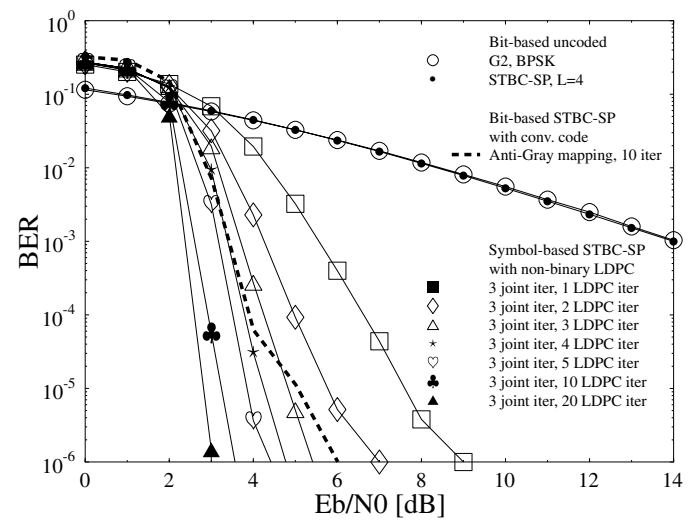

Figure 4: Performance comparison of symbol-based non-binary LDPCcoded STBC-SP schemes using a fixed number of joint iterations and various numbers of LDPC iterations against both an identical-throughput 1BPS uncoded STBC-SP scheme [5], Alamouti's conventional $G_{2}$ BPSK scheme [2] and against bit-based convolutional-coded STBC-SP schemes [8] employing Gray Mapping (GM) and Anti-Gray Mapping (AGM), when communicating over a correlated Rayleigh fading channel having $f_{D}=0.1$.

\section{CONCLUSION}

In this paper we proposed a novel symbol-based iterative system that exploits the advantages of both non-binary LDPC codes [15] as well as those of the STBC-SP scheme of [5]. Our investigations demonstrated that significant performance improvements may be achieved by the proposed scheme over established orthogonal STBC designs, constituted by the STBC-SP scheme of [5] as well as over the bit-based turbo-detected STBC-SP scheme of [8]. The achievable performance was also investigated by illustrating the coding gain attained, when different combinations of external joint and internal LDPC iterations are used. Our future research includes the use of a rate-1 inner precoder $[17,18]$ and the employment of symbol-based Extrinsic Information Transfer (EXIT) Charts [16] to find more beneficial LDPC generator matrices that may lead to lower required $E_{b} / N_{0}$ values. Additionally, another novel scheme is currently investigated that employs the bit-based turbo-detected STBC-SP scheme of [8] as an inner code, while the non-binary LDPC is incorporated as an outer code and performing symbol-based outer iterative decoding and bit-based inner turbodetection in conjunction with iterative demapping.

\section{REFERENCES}

[1] L. Hanzo, T. H. Liew, and B. L. Yeap, Turbo Coding, Turbo Equalisation and Space-Time Coding: for Transmission over Fading Channels. Chichester, England: John Wiley and Sons Ltd and IEEE Press, NY, USA, 2002.

[2] S. Alamouti, "A simple transmit diversity technique for wireless communications," IEEE Journal on Selected Areas in Communications, vol. 16, no. 8, pp. 1451-1458, 1998.

[3] V. Tarokh, H. Jafarkhani, and A. Calderbank, "Space-time block codes from orthogonal designs," IEEE Transactions on Information Theory, vol. 45, pp. 1456-1467, Jul 1999.

[4] V. Tarokh, H. Jafarkhani, and A. Calderbank, "Space-time block coding for wireless communications: performance results," IEEE Journal on Selected Areas in Communications, vol. 17, pp. 451-460, Mar 1999.

[5] W. Su, Z. Safar, and K. J. R. Liu, "Space-time signal design for timecorrelated Rayleigh fading channels," in IEEE International Confer-

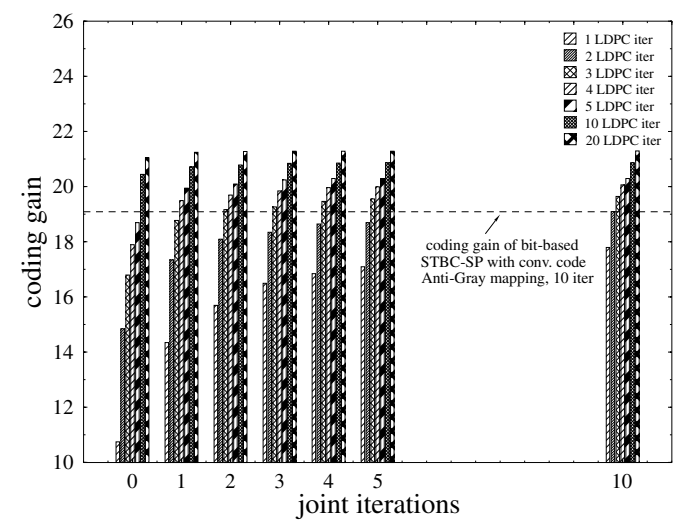

Figure 5: Coding gain of various symbol-based non-binary LDPC-coded STBC-SP schemes in conjunction with different combinations of joint and LDPC iterations as compared to both the identical-throughput 1BPS uncoded STBC-SP scheme of [5], Alamouti's conventional $G_{2}$-BPSK scheme [2] and to the bit-based convolutional-coded STBC-SP scheme [8] employing Anti-Gray Mapping, when communicating over a correlated Rayleigh fading channel having $f_{D}=0.1$.

ence on Communications, vol. 5, (Anchorage, Alaska), pp. 31753179, 2003.

[6] W. Su and X. G. Xia, "On space-time block codes from complex orthogonal designs," Wireless Personal Communications, (Kluwer Academic Publishers), vol. 25, pp. 1-26, April 2003.

[7] J. H. Conway and N. J. Sloane, Sphere Packings, Lattices and Groups. Springer-Verlag, 1999.

[8] O. Alamri, B. L. Yeap, and L. Hanzo, "Turbo detection of channelcoded space-time signals using sphere packing modulation," in IEEE Vehicular Technology Conference, vol. 4, (Los Angeles, USA), pp. 2498-2502, Sep 2004.

[9] S. ten Brink, J. Speidel, and R.-H. Yan, "Iterative demappping and decoding for multilevel modulation," in IEEE Global Telecommunications Conference, vol. 1, (Sydney, Australia), pp. 579-584, 8-12 Nov 1998.

[10] R. Gallager, "Low density parity check codes," IEEE Transactions on Information Theory, vol. 8, pp. 21-28, Jan. 1962.

[11] C. Berrou, A. Glavieux, and P. Thitimajshima, "Near Shannon limit error-correcting coding and decoding: Turbo codes," Proceedings of the IEEE International Confrence on Communications, pp. 10641070, May 1993.

[12] T. Richardson and R. Urbanke, "The renaissance of Gallager's low-density parity-check codes," IEEE Communications Magazine, vol. 41, pp. 126-131, Aug 2003.

[13] D. J. C. MacKay and R. M. Neal, "Near Shannon limit performance of low density parity check codes," Electronics Letters, vol. 33, pp. 457-458, 13 March 997.

[14] R. U. T. Richardson, "The capacity of low-density parity check codes under message-passing decoding," IEEE Transaction on Information Theory, vol. 47, pp. 599-618, Feb 2001.

[15] M. C. Davey and D. J. C. MacKay, "Low density parity check codes over GF(q)," IEEE Communications Letters, vol. 2, pp. 165-167, June 1998

[16] A. Grant, "Convergence of non-binary iterative decoding," IEEE Global Telecommunications Conference, vol. 2, pp. 1058-1062, Nov 2001.

[17] D. Divsalar, S. Dolinar, and F. Pollara, "Serial concatenated trellis coded modulation with rate-1 inner code," in IEEE Global Telecommunications Conference, vol. 2, pp. 777-782, 27 Nov-1 Dec 2000.

[18] L. Lifang, D. Divsalar, and S. Dolinar, "Iterative demodulation, demapping, and decoding of coded non-square QAM," in IEEE Transactions on Communications, vol. 53, pp. 16-19, Jan 2005. 